\title{
Explicit Causal Recursive Estimators for Continuous-Time Bivariate Markov Chains
}

\author{
Brian L. Mark, Senior Member, IEEE, and Yariv Ephraim, Fellow, IEEE
}

\begin{abstract}
A bivariate Markov chain comprises a pair of random processes which are jointly Markov. In this paper, both processes are assumed to be continuous-time with finite state space. One of the two processes is observable, while the other is an underlying process which affects the statistical properties of the observable process. Neither the observable, nor the underlying process, is required to be a Markov chain. Examples of bivariate Markov chains include the Markov modulated Markov process (MMMP), the Markov modulated Poisson process (MMPP), and the batch Markovian arrival process (BMAP). We develop explicit causal recursions for estimating the number of jumps from one state to another, and the total sojourn time in each state, of a general bivariate Markov chain. Explicit causal recursions of these statistics were previously developed for the MMMP and the MMPP using the transformation of measure approach. We argue that this approach cannot be extended to a general bivariate Markov chain. Instead, we modify the approach developed by Rydén for noncausal estimation of the same statistics of an MMPP, and use the state augmentation approach of Zeitouni and Dembo and a matrix recursion from Stiller and Radons, to derive the causal recursions. The new recursions do not require any numerical integration or sampling scheme of the continuous-time bivariate Markov chain.
\end{abstract}

Index Terms-Markov processes, recursive estimation.

\section{INTRODUCTION}

A bivariate Markov chain comprises a pair of random processes which are jointly Markov. In this paper, we focus on bivariate Markov chains for which the pair of random processes is finite-state and continuous-time. Each of the two processes comprising the bivariate Markov chain need not be Markov, and the two processes may jump simultaneously. Only one of these two processes is assumed observable. The distribution of the dwell-time of the observable process in each state is phase-type [22]. Important examples of bivariate Markov chains include the Markov modulated Poisson process (MMPP) and its Markov modulated Markov process (MMMP) generalization, see, e.g., [16], [17], [29], [31], and the Markovian arrival process (MAP) and its generalization in the form

Manuscript received July 11, 2013; revised January 28, 2014; accepted March 24, 2014. Date of publication March 31, 2014; date of current version April 25,2014 . The associate editor coordinating the review of this manuscript and approving it for publication was Prof. Minyue Fu. This work was supported in part by the U.S. National Science Foundation under Grant CCF-0916568.

The authors with the Department of Electrical and Computer Engineering, George Mason University, Fairfax, VA 22030 USA (e-mail: bmark@gmu.edu; yephraim@gmu.edu).

Color versions of one or more of the figures in this paper are available online at http://ieeexplore.ieee.org.

Digital Object Identifier 10.1109/TSP.2014.2314434 of a batch Markovian arrival process (BMAP), see, e.g., [5], [20], [21]. Note that while the MMPP, MAP and BMAP have countably infinite state spaces, they can be represented without loss of generality as finite-state processes when modulo counts are used. Bivariate Markov chains are common models arising in applications such as the study of ion channel currents [2], [4], [8], [27], traffic modeling [17], [20], network congestion [33], [38], spectrum sensing in cognitive radio networks [19], [24], [25], [35], and phylogenetics [6], [18], [26].

Bivariate Markov chains also occur in discrete time. When the underlying process of a discrete-time bivariate Markov chain is Markov, and the observable process comprises a sequence of conditionally independent random variables, then the bivariate Markov chain becomes a finite-alphabet hidden Markov model (HMM). The dwell-time of the observable process of the bivariate Markov chain in each of its states has a discrete phase-type distribution while the dwell-time of the observable process of an HMM has a geometric distribution. Another important relation exists between certain types of continuous-time finite-state bivariate Markov chains and HMMs. For example, the MMPP has an HMM representation, as was first shown in [31]. A thorough review of bivariate Markov processes, in discrete and continuous time, with finite as well as continuous alphabet, may be found in [13]. In this paper, we exclusively focus on finite-state continuous-time bivariate Markov chains which, for brevity, we shall simply refer to as "bivariate Markov chains."

Given a sequence of observations from a process modeled by a bivariate Markov chain, it is frequently of interest to estimate three key statistics: the state, the number jumps from one state to another, and the total sojourn time in each state. An estimate of the average sojourn time in a given state can essentially be obtained as a ratio of the total sojourn time in the state and the number of jumps out of the state. Efficient online estimators of these key statistics are needed in real-time applications of bivariate Markov chain models. In the network congestion application, for example, a path in a network is modeled as a bivariate Markov chain wherein one or more of the underlying states indicates a certain congestion level (cf. [38]). Real-time routing decisions based on the mean time in a congested state or subset of states need to be executed in an online fashion. A key feature of causal recursive estimators is that estimation of the statistics of the process, given its parameter, can be performed online, without the need to store the entire training data. In the cognitive radio application, frequency channels are modeled by a bivariate Markov chain with an underlying chain representing either the active or idle state of a primary system (cf. [25]). Here, 
a real-time decision on which channel a cognitive secondary system should access could be based on a causal recursive estimator of the mean sojourn time in the active state.

In this paper, we derive explicit causal recursive estimators for the number of jumps from one state to another and the total sojourn time in each state of the bivariate Markov chain. The recursions are explicit in the sense that they do not require numerical integration or sampling of the continuous-time bivariate Markov chain. We also specialize the recursions for the MMMP and the MAP. An explicit causal recursion for estimating the state of the bivariate Markov chain was developed earlier in [30], [39]. Previously, explicit causal recursions for the number of jumps and the total sojourn time had been developed only for the MMMP and the MMPP [11] using the transformation of measure approach. The latter approach was used earlier in [9] to derive causal recursions for the MMMP in the form of stochastic integrals. Recursions of a similar form were developed for the MMPP and implemented via a sampling scheme in [10]. The transformation of measure technique is not applicable to the general bivariate Markov chain, since the underlying and observable processes may jump simultaneously, and hence, cannot be independent under any reference measure [12].

The derivation of the explicit causal recursions is based on our earlier derivation of forward-backward or non-causal recursions in [22], which in turn is based on Rydén's earlier derivation for the MMPP in [32]. We follow that derivation and show how it can be modified to provide the causal recursions. We rely on the powerful state augmentation approach of [40], on an expansion of the backward recursion as stated in [34], and on the work of [37] for evaluating integrals of matrix exponentials. In the state augmentation approach, the statistic of interest is jointly estimated along with the state of the underlying unobservable process. This approach was used in [40] for estimating the number of jumps of a Markov chain observed in white noise, and was later adopted in [9], [12], and [11].

The remainder of the paper is organized as follows. In Section II, we define the continuous-time bivariate Markov chain and discuss some of its key properties. The explicit causal recursive estimators for the bivariate Markov chain are developed in Section III. Specializations of these recursions for the MMMP, MAP, and MMPP are presented in Section IV. In Section $V$, a numerical example demonstrating the performance of the causal recursive estimators is presented. Concluding remarks are provided in Section VI.

\section{Continuous-Time Bivariate Markov Chain}

Consider a finite-state, homogeneous, separable, continuoustime bivariate Markov chain

$$
Z=(X, S)=\{(X(t), S(t)), t \geq 0\},
$$

defined on a probability space. We assume, without loss of generality, that the state space of $X$ is given by $\mathbb{X}=\{1, \ldots, d\}$. For each state $a \in \mathbb{X}$, we assume that $S$ takes values in $\mathbb{S}_{a}=$ $\left\{1, \ldots, r_{a}\right\}$. The orders $d$ and $r_{1}, \ldots, r_{d}$ are assumed known. The state space of $Z$ is then given by

$$
\mathbb{Z}=\bigcup_{a=1}^{d}\left\{(a, i), i \in \mathbb{S}_{a}\right\} .
$$

Let $r=\max _{a \in \mathbb{X}} r_{a}$. Note that $\mathbb{Z}$ is a subset of $\{1, \ldots, d\} \times$ $\{1, \ldots, r\}$. The process $X$ is assumed observable, while the process $S$ is the underlying process. Such a bivariate Markov chain may be obtained by applying an aggregating deterministic function to a Markov chain [13], [22]. The processes $X$ and $S$ may jump simultaneously. In addition, neither $X$ nor $S$ need be Markov. With probability one, all sample paths of $Z$ are rightcontinuous step functions with a finite number of jumps in any finite interval [1, Theorem 2.1].

The bivariate Markov chain $Z$ is parameterized by a generator matrix

$$
G=\left\{g_{a b}(i j), a, b \in \mathbb{X} ; i \in \mathbb{S}_{a}, j \in \mathbb{S}_{b}\right\},
$$

where the set of joint states $\{(a, i)\}$ is ordered lexicographically. With $P(\cdot)$ denoting the probability measure on the given space,

$$
g_{a b}(i j)=\lim _{\epsilon \rightarrow 0} \frac{1}{\epsilon} P(Z(t+\epsilon)=(b, j) \mid Z(t)=(a, i)),
$$

for $(a, i) \neq(b, j)$ and $g_{a a}(i i)=-\sum_{(b, j) \neq(a, i)} g_{a b}(i j)$. The generator matrix can be expressed as a block matrix $G=\left\{G_{a b}, a, b \in \mathbb{X}\right\}$, where $G_{a b}=\left\{g_{a b}(i j), i \in \mathbb{S}_{a}, j \in \mathbb{S}_{b}\right\}$ are $r_{a} \times r_{b}$ matrices. We assume that $G$ and each of its block diagonal submatrices $G_{a a}, a \in \mathbb{X}$, are irreducible.

As an example of two non-Markovian processes that are jointly Markov, consider the following situation. Let $Z$ be a Markov chain with state space $\{1,2,3\}$. Let $X=g_{1}(Z)$ where $X=1$ if $Z=1$ and $X=2$ when $Z \in\{2,3\}$. Let $S=g_{2}(Z)$ where $S=2$ if $Z \in\{1,2\}$ and $S=3$ when $Z=3$. The functions $g_{1}$ and $g_{2}$ are aggregating functions and hence neither $X$ nor $S$ is Markov, see, e.g., [7]. However, $(X, S)$ is in a $1-1$ correspondence with $Z$ and hence is Markov. Aggregated Markov chains are often encountered in many practical applications such as in ion-channel modeling, see, e.g., [2], [4], [8], [28].

\section{A. Density of Observable Process}

Assume that the observable process $X$ of a bivariate Markov chain $Z=(X, S)$ jumps at $T_{0}<T_{1}<T_{2}<\cdots<T_{n}<\cdots$ where $T_{0}=0$ is an arbitrary time origin. Let $X_{k}=X\left(T_{k}\right)$ denote the state of $X$ in the interval $\left[T_{k}, T_{k+1}\right)$ for $k=0,1,2, \ldots$ Define $S_{k}=S\left(T_{k}\right)$ to be the state of $S$ at the jump time $T_{k}$ of $X$. Let $Z_{k}=Z\left(T_{k}\right)=\left(X_{k}, S_{k}\right)$. For $k=1,2, \ldots$, let $\Delta T_{k}=T_{k}-T_{k-1}$ denote the dwell-time of $X$ in state $X_{k-1}$. It was shown in [14, Lemma 3] that the sampled bivariate Markov chain $\left\{Z_{k}\right\}$ has a single irreducible set of states which may be periodic. The remaining states are transient. Hence, $\left\{Z_{k}\right\}$ has a unique stationary distribution, with zero entries for the transient states. Throughout the paper, we denote realizations of $X_{k}, S_{k}$, $Z_{k}, T_{k}$, and $\Delta T_{k}$ by $x_{k}, s_{k}, z_{k}, t_{k}$, and $\Delta t_{k}$, respectively.

The process $\left\{Z_{k}, T_{k}\right\}$ is a Markov renewal process [22]. Define for any $(a, i),(b, j) \in \mathbb{Z}$,

$$
F_{i j}^{a b}(t)=P\left(Z_{1}=(b, j), \Delta T_{1} \leq t \mid Z_{0}=(a, i)\right) .
$$

By homogeneity of the bivariate Markov chain,

$$
F_{i j}^{a b}(t)=P\left(Z_{k+1}=(b, j), \Delta T_{k+1} \leq t \mid Z_{k}=(a, i)\right)
$$


for all $k$ 's. The semi-Markov kernel of $\left\{Z_{k}, T_{k}\right\}$ is given by

$$
\left\{F_{i j}^{a b}(t): a, b \in \mathbb{X} ; i \in \mathbb{S}_{a} ; j \in \mathbb{S}_{b} ; t \geq 0\right\} .
$$

The derivative of $F_{i j}^{a b}(t)$ with respect to $t$ gives the transition density $f_{i j}^{a b}(t)$. Let $f^{a b}(t)=\left\{f_{i j}^{a b}(t), i \in \mathbb{S}_{a}, j \in \mathbb{S}_{b}\right\}$ denote the transition density matrix associated with the transition of $X$ from $a$ to $b$. We are also interested in the transition probability

$$
\bar{F}_{i j}^{a}(t)=P\left(S(t)=j, \Delta T_{1}>t \mid X(0)=a, S(0)=i\right)
$$

and its transition matrix $\bar{F}^{a}(t)=\left\{\bar{F}_{i j}^{a}(t), i, j \in \mathbb{S}_{a}\right\}$. Explicit forms for $f^{a b}(t)$ and $\bar{F}^{a}(t)$ were derived in [8], [22], [27] as follows:

$$
f^{a b}(t)=e^{G_{a a} t} G_{a b}, a \neq b
$$

and

$$
\bar{F}^{a}(t)=e^{G_{a a} t}, t \geq 0 .
$$

To simplify notation in the sequel, we shall use $P(\cdot)$ to denote probability as well as a density, as appropriate (cf. [32]). The exact meaning of expressions involving $P(\cdot)$ should be clear from the context. The density of $\left\{X(t), 0 \leq t \leq t_{n}\right\}$ can be expressed in terms of the initial distribution of $Z$ and the transition density matrix $f^{a b}(t)$. For $X_{0}=x_{0}$ and $S_{0}=i$, let $\nu_{x_{0}}(i)=$ $P\left(Z_{0}=\left(x_{0}, i\right)\right)$ and let $\nu_{x_{0}}=\left\{\nu_{x_{0}}(1), \ldots, \nu_{x_{0}}\left(r_{x_{0}}\right)\right\}$. From the Markov renewal property, the density of the sample path is obtained from the density of $\left\{x_{0}, t_{1}, x_{1}, \ldots, t_{n}, x_{n}\right\}$ and is given by

$$
P\left(X(t), 0 \leq t \leq t_{n}\right)=\nu_{x_{0}}\left\{\prod_{k=1}^{n} f^{x_{k-1} x_{k}}\left(\Delta t_{k}\right)\right\} \mathbf{1},
$$

where 1 denotes a column vector of all ones. Extension of the likelihood (11) to an intermediate time instant $\tau \in\left(t_{k}, t_{k+1}\right)$ is also possible by using $\bar{F}^{a}\left(\tau-t_{k}\right)$. The observable sequence $\left\{\left(X_{k}, \Delta T_{k}\right)\right\}$ is stationary when $\nu_{x_{0}}$ is a member of the stationary distribution $\nu=\left\{\nu_{1}, \ldots, \nu_{d}\right\}$ of the sampled bivariate Markov chain $\left\{Z_{k}\right\}$ for any $x_{0} \in \mathbb{X}$.

\section{EXPLicit CAUSAL ReCURSIONS}

In this section, we develop explicit causal recursions for estimating the state, the number of jumps, and the total sojourn time for the bivariate Markov chain. The recursion for the state was originally developed in [30], [39]. It is given here in terms of our notation and the context of this paper.

\section{A. State Recursion}

The conditional mean estimate of $S(t)$ given $\{X(\tau), 0 \leq$ $\tau \leq t\}$ can be obtained from the conditional mean estimates of the indicator functions

$$
\varphi_{i}(t)= \begin{cases}1, & S(t)=i \\ 0, & \text { otherwise }\end{cases}
$$

for $i \in \mathbb{S}_{x_{t}}$. When $t$ is a jump point of $X$, i.e., $t=t_{k}, k=$ $0,1, \ldots$, the conditional mean estimate of $\varphi_{i}\left(t_{k}\right), i \in \mathbb{S}_{x_{k}}$, is given by $P\left(S_{k}=i \mid X(\tau), 0 \leq \tau \leq t_{k}\right)$, and it can be obtained by scaling the forward density $P\left(S_{k}=i, X(\tau), 0 \leq \tau \leq t_{k}\right)$. The vector of forward densities, for all $i \in \mathbb{S}_{x_{k}}$, follows recursively from the product of the first $k$ factors in (11). Define the row vector $\varphi\left(t_{k}\right)=\left\{\varphi_{i}\left(t_{k}\right), i \in \mathbb{S}_{x_{k}}\right\}$, and let $\tilde{L}(k)=$ $E\left\{\boldsymbol{\varphi}\left(t_{k}\right) \mid X(\tau), 0 \leq \tau \leq t_{k}\right\}$. We have

$$
\begin{aligned}
\tilde{L}(0) & =\frac{\nu_{x_{0}}}{c_{0}}, \\
\tilde{L}(k) & =\frac{\tilde{L}(k-1) f^{x_{k-1} x_{k}}\left(\Delta t_{k}\right)}{c_{k}}, k=1,2, \ldots,
\end{aligned}
$$

where

$$
\begin{aligned}
& c_{0}=\nu_{x_{0}} \mathbf{1}, \\
& c_{k}=\tilde{L}(k-1) f^{x_{k-1} x_{k}}\left(\Delta t_{k}\right) \mathbf{1}, k=1,2, \ldots,
\end{aligned}
$$

are the scaling constants. The scaled causal state recursions as defined by (13) and (14) are equivalent to (26) and (27) in [22]. ${ }^{1}$ As a byproduct, we can write the likelihood of $X$ as (cf. Equation (11))

$$
P\left(X(\tau), 0 \leq \tau \leq t_{n}\right)=\prod_{k=0}^{n} c_{k} .
$$

\section{B. Number of Jumps Recursion}

Define the indicator function

$$
\chi_{a i}(t)= \begin{cases}1, & Z(t)=(a, i), \\ 0, & \text { otherwise. }\end{cases}
$$

The number of jumps of the bivariate Markov chain $Z$, from state $(a, i)$ to state $(b, j)$ in $[0, t]$, where $(a, i) \neq(b, j)$, is given by

$$
m_{i j}^{a b}(t)=\sum_{0 \leq \tau \leq t} \chi_{a i}(\tau-) \chi_{b j}(\tau)
$$

where the sum is over the jump points of $Z$ in $[0, t]$. Following the state augmentation approach of [40], define the row vector

$$
\hat{\boldsymbol{m}}_{i j}^{a b}(t)=E\left\{m_{i j}^{a b}(t) \boldsymbol{\varphi}(t) \mid X(\xi), 0 \leq \xi \leq t\right\},
$$

and note that the conditional mean estimate of the number of jumps is given by $\hat{\boldsymbol{m}}_{i j}^{a b}(t) \mathbf{1}$. Hence, we need only derive a causal recursion for $\hat{\boldsymbol{m}}_{i j}^{a b}(t)$. Let $\mathbf{1}_{l}$ denote a column vector with a one in the $l$ th element and zeros elsewhere. Using (17), the $l$ th component of $\hat{\boldsymbol{m}}_{i j}^{a b}(t)$, i.e., $\hat{\boldsymbol{m}}_{i j}^{a b}(t) \mathbf{1}_{l}$, is given by

$$
\begin{array}{r}
{\left[\hat{\boldsymbol{m}}_{i j}^{a b}(t)\right]_{l}=\sum_{0 \leq \tau \leq t} P(Z(\tau-)=(a, i), Z(\tau)=(b, j),} \\
S(t)=l \mid X(\xi), 0 \leq \xi \leq t) .
\end{array}
$$

This expression is evaluated in Propositions 1 and 2 below for two separate cases, first when $\{a=b, i \neq j\}$, and then for $a \neq b$, regardless of whether or not the underlying chain also jumps at the same time, i.e., regardless of the values of $i$ and $j$. For the first case, the following integral arises.

$$
\mathcal{K}_{i j}(k)=\int_{t_{k}}^{t_{k+1}} \tilde{L}(k) \bar{F}^{x_{k}}\left(\tau-t_{k}\right) \mathbf{1}_{i} \mathbf{1}_{j}^{\prime} f^{x_{k} x_{k+1}}\left(t_{k+1}-\tau\right) d \tau,
$$

${ }^{1}$ In [22, Eq. (26)], $c_{0}$ should be defined as $c_{0}=\nu_{x_{0}} \mathbf{1}$, and the products of the scaling constants starting from $c_{1}$ in the subsequent (29), (31), and (32), should instead start from $c_{0}$. 
where' denotes matrix transpose. A similar integral was encountered in [22]. Each $\mathcal{K}_{i j}(k)$ can be computed as follows:

$$
\begin{aligned}
& \int_{t_{k}}^{t_{k+1}}\left[\tilde{L}(k) \bar{F}^{x_{k}}\left(\tau-t_{k}\right) \mathbf{1}_{i}\right]^{\prime} \mathbf{1}_{j}^{\prime} f^{x_{k} x_{k+1}}\left(t_{k+1}-\tau\right) d \tau \\
= & \mathbf{1}_{i}^{\prime} \int_{t_{k}}^{t_{k+1}}\left[\bar{F}^{x_{k}}\left(\tau-t_{k}\right)\right]^{\prime} \tilde{L}(k)^{\prime} \mathbf{1}_{j}^{\prime} f^{x_{k} x_{k+1}}\left(t_{k+1}-\tau\right) d \tau \\
= & \mathbf{1}_{i}^{\prime} \int_{t_{k}}^{t_{k+1}} e^{G_{x_{k} x_{k}}^{\prime}\left(\tau-t_{k}\right)} \tilde{L}(k)^{\prime} \mathbf{1}_{j}^{\prime} e^{G_{x_{k} x_{k}}\left(t_{k+1}-\tau\right)} d \tau G_{x_{k} x_{k+1}} .
\end{aligned}
$$

From [37], we have

$$
\mathcal{K}_{i j}(k)=\mathbf{1}_{i}^{\prime}\left[e^{C_{k}^{j} \Delta t_{k+1}}\right]_{21} G_{x_{k} x_{k+1}},
$$

where

$$
C_{k}^{j}=\left[\begin{array}{cc}
G_{x_{k} x_{k}} & \mathbf{0} \\
\tilde{L}(k)^{\prime} \mathbf{1}_{j}^{\prime} & G_{x_{k} x_{k}}^{\prime}
\end{array}\right]
$$

and $[\cdot]_{21}$ denotes the lower left block of the referenced matrix. Note that the definition of $C_{k}^{j}$ is similar to that given in [11, Eq. (48)] for the MMMP.

For both cases, we will also find it convenient to define

$$
\tilde{M}(k, n)=\prod_{m=k}^{n} \frac{f^{x_{m-1} x_{m}}\left(\Delta t_{m}\right)}{c_{m}}, 1 \leq k \leq n+1,
$$

for $n=0,1,2, \ldots$, with $\tilde{M}(k+1, k)=I$. A causal recursion for $\tilde{M}(k, n)$ in the second variable $n$, where $n \geq k$, is given as follows:

$$
\tilde{M}(k, n)=\tilde{M}(k, n-1) \frac{f^{x_{n-1} x_{n}}\left(\Delta t_{n}\right)}{c_{n}} .
$$

Proposition 1: $\quad[a=b, i \neq j]$

$$
\begin{aligned}
& \hat{\boldsymbol{m}}_{i j}^{a a}\left(t_{n}\right)=\frac{1}{c_{n}}\left\{\hat{\boldsymbol{m}}_{i j}^{a a}\left(t_{n-1}\right) e^{G_{x_{n-1} x_{n-1}} \Delta t_{n}}\right. \\
& \left.\quad+I_{\left\{x_{n-1}=a\right\}} g_{a a}(i j) \mathbf{1}_{i}^{\prime}\left[e^{C_{n-1}^{j} \Delta t_{n}}\right]_{21}\right\} G_{x_{n-1} x_{n}},
\end{aligned}
$$

for $n \geq 1$, with the initialization $\hat{\boldsymbol{m}}_{i j}^{a a}\left(t_{0}\right)=\mathbf{0}$.

Proof: From [22, Eq. (49)],

$$
\begin{array}{r}
{\left[\hat{\boldsymbol{m}}_{i j}^{a a}(t)\right]_{l}=\int_{0}^{t} P(Z(\tau-)=(a, i), Z(\tau)=(a, j),} \\
S(t)=l \mid X(\xi), 0 \leq \xi \leq t) d \tau .
\end{array}
$$

Suppose $\tau \in\left[t_{k}, t_{k+1}\right)$ and $x_{k}=a$. Applying (13) and (15) to the integrand of (27) with $t=t_{n}$, we obtain

$$
\begin{aligned}
& P\left(Z(\tau-)=(a, i), Z(\tau)=(a, j), S\left(t_{n}\right)=l \mid X(\xi), 0 \leq \xi \leq t_{n}\right) \\
& =\frac{P\left(Z(\tau-)=(a, i), Z(\tau)=(a, j), S\left(t_{n}\right)=l, X(\xi), 0 \leq \xi \leq t_{n}\right)}{P\left(X(\xi), 0 \leq \xi \leq t_{n}\right)} \\
& =\frac{\left\{\nu_{x_{0}} \prod_{m=1}^{k} f^{x_{m-1} x_{m}}\left(\Delta t_{m}\right)\right\}}{P\left(X(\xi), 0 \leq \xi \leq t_{n}\right)} \bar{F}^{x_{k}}\left(\tau-t_{k}\right) \mathbf{1}_{i} g_{x_{k} x_{k}}(i j) \mathbf{1}_{j}^{\prime} \\
& \quad \cdot f^{x_{k} x_{k+1}\left(t_{k+1}-\tau\right)\left\{\prod_{m=k+2}^{n} f^{x_{m-1} x_{m}}\left(\Delta t_{m}\right)\right\} \mathbf{1}_{l}=} \\
& \frac{g_{a a}(i j) \tilde{L}(k)}{c_{k+1}} \bar{F}^{x_{k}}\left(\tau-t_{k}\right) \mathbf{1}_{i} \mathbf{1}_{j}^{\prime} f^{x_{k} x_{k+1}\left(t_{k+1}-\tau\right) \tilde{M}(k+2, n) \mathbf{1}_{l} .}
\end{aligned}
$$

From (27), we have

$$
\begin{gathered}
{\left[\hat{\boldsymbol{m}}_{i j}^{a a}\left(t_{n}\right)\right]_{l}=\sum_{\substack{k=0 \\
x_{k}=a}}^{n-1} \int_{t_{k}}^{t_{k+1}} P(Z(\tau-)=(a, i), Z(\tau)=(a, j),} \\
\left.S\left(t_{n}\right)=l \mid X(\xi), 0 \leq \xi \leq t_{n}\right) d \tau
\end{gathered}
$$

Substituting (28) into (29) and applying (21), it follows that

$$
\left[\hat{\boldsymbol{m}}_{i j}^{a a}\left(t_{n}\right)\right]_{l}=\sum_{\substack{k=0 \\ x_{k}=a}}^{n-1} \frac{g_{a a}(i j)}{c_{k+1}} \mathcal{K}_{i j}(k) \tilde{M}(k+2, n) \mathbf{1}_{l}
$$

Hence,

$$
\hat{\boldsymbol{m}}_{i j}^{a a}\left(t_{n}\right)=\sum_{\substack{k=0 \\ x_{k}=a}}^{n-1} \frac{g_{a a}(i j)}{c_{k+1}} \mathcal{K}_{i j}(k) \tilde{M}(k+2, n)
$$

Using (25), Equation (31) can be recast in the form of a causal recursion as follows:

$$
\begin{aligned}
\hat{\boldsymbol{m}}_{i j}^{a a}\left(t_{n}\right)= & \sum_{\substack{k=0 \\
x_{k}=a}}^{n-2} \frac{g_{a a}(i j)}{c_{k+1}} \mathcal{K}_{i j}(k) \tilde{M}(k+2, n) \\
& \quad+I_{\left\{x_{n-1}=a\right\}} \frac{g_{a a}(i j)}{c_{n}} \mathcal{K}_{i j}(n-1) \\
= & \hat{\boldsymbol{m}}_{i j}^{a a}\left(t_{n-1}\right) \frac{f^{x_{n-1} x_{n}}\left(\Delta t_{n}\right)}{c_{n}} \\
& \quad+I_{\left\{x_{n-1}=a\right\}} \frac{g_{a a}(i j)}{c_{n}} \mathcal{K}_{i j}(n-1) .
\end{aligned}
$$

Applying (9) and (22) in (32), we obtain (26).

For the second case, where $a \neq b$, the sum in (19) can be confined to the jump points of the observable process $X$ from state $a$ to state $b$, irrespective of jumps of $S$. Hence,

$$
\begin{array}{r}
{\left[m_{i j}^{a b}(t)\right]_{l}=\sum_{\substack{k=0 \\
\left(x_{k}, x_{k+1}\right)=(a, b)}}^{n-1} P\left(Z\left(t_{k}-\right)=(a, i), Z\left(t_{k}\right)=(b, j),\right.} \\
S(t)=l \mid X(\xi), 0 \leq \xi \leq t) .
\end{array}
$$

Proposition 2: $[a \neq b]$

$$
\begin{aligned}
& \hat{\boldsymbol{m}}_{i j}^{a b}\left(t_{n}\right)=\frac{1}{c_{n}}\left\{\hat{\boldsymbol{m}}_{i j}^{a b}\left(t_{n-1}\right) e^{G_{x_{n-1} x_{n-1}} \Delta t_{n}} G_{x_{n-1} x_{n}}\right. \\
& \left.+I_{\left\{x_{n-1}=a, x_{n}=b\right\}} g_{a b}(i j) \tilde{L}(n-1) e^{G_{x_{n-1} x_{n-1}} \Delta t_{n}} \mathbf{1}_{i} \mathbf{1}_{j}^{\prime}\right\}
\end{aligned}
$$

for $n \geq 1$, with the initialization $\hat{\boldsymbol{m}}_{i j}^{a b}\left(t_{0}\right)=\mathbf{0}$. 
Proof: Suppose $k$ is such that $x_{k}=a$ and $x_{k+1}=b$. Similarly to Proposition 1,2

$$
\begin{aligned}
& P\left(Z\left(t_{k+1}-\right)=(a, i), Z\left(t_{k+1}\right)=(b, j), S\left(t_{n}\right)=l\right. \\
= & \frac{\left.\mid X(\xi), 0 \leq \xi \leq t_{n}\right)}{P\left(X(\xi), 0 \leq \xi \leq t_{n}\right)} \cdot P\left(Z\left(t_{k+1}-\right)=(a, i),\right. \\
\left.Z\left(t_{k+1}\right)=(b, j), S\left(t_{n}\right)=l, X(\xi), 0 \leq \xi \leq t_{n}\right) & \frac{\nu_{x_{0}} \prod_{m=1}^{k} f^{x_{m-1} x_{m}}\left(\Delta t_{m}\right)}{P\left(X(\xi), 0 \leq \xi \leq t_{n}\right)} \bar{F}^{x_{k}}\left(\Delta t_{k+1}\right) \mathbf{1}_{i} g_{x_{k} x_{k+1}}(i j) \mathbf{1}_{j}^{\prime} \\
& \cdot\left\{\prod_{m=k+2}^{n} f^{x_{m-1} x_{m}}\left(\Delta t_{m}\right)\right\} \mathbf{1}_{l} \\
= & \frac{g_{a b}(i j)}{c_{k+1}} \tilde{L}(k) \bar{F}^{x_{k}}\left(\Delta t_{k+1}\right) \mathbf{1}_{i} \mathbf{1}_{j}^{\prime} \tilde{M}(k+2, n) \mathbf{1}_{l} .
\end{aligned}
$$

Substituting (35) into (33), we have

$$
\begin{aligned}
& {\left[\hat{\boldsymbol{m}}_{i j}^{a b}\left(t_{n}\right)\right]_{l}=} \\
& \sum_{\substack{k=0: x_{k}=a \\
x_{k+1}=b}}^{n-1} \frac{g_{a b}(i j)}{c_{k+1}} \tilde{L}(k) \bar{F}^{x_{k}}\left(\Delta t_{k+1}\right) \mathbf{1}_{i} \mathbf{1}_{j}^{\prime} \tilde{M}(k+2, n) \mathbf{1}_{l} .
\end{aligned}
$$

Hence,

$$
\begin{aligned}
& \hat{\boldsymbol{m}}_{i j}^{a b}\left(t_{n}\right) \\
& =\sum_{\substack{k=0: x_{k}=a \\
x_{k+1}=b}}^{n-1} \frac{g_{a b}(i j)}{c_{k+1}} \tilde{L}(k) \bar{F}^{x_{k}}\left(\Delta t_{k+1}\right) \mathbf{1}_{i} \mathbf{1}_{j}^{\prime} \tilde{M}(k+2, n) .
\end{aligned}
$$

Applying the causal recursions (13) and (25), Equation (37) can be recast in the form of a causal recursion as follows:

$$
\begin{aligned}
& \hat{\boldsymbol{m}}_{i j}^{a b}\left(t_{n}\right) \\
= & \sum_{\substack{k=0: x_{k}=a \\
x_{k+1}=b}}^{n-2} \frac{g_{a b}(i j)}{c_{k+1}} \tilde{L}(k) \bar{F}^{x_{k}}\left(\Delta t_{k+1}\right) \mathbf{1}_{i} \mathbf{1}_{j}^{\prime} \tilde{M}(k+2, n) \\
& +I_{\left\{x_{n-1}=a, x_{n}=b\right\}} \frac{g_{a b}(i j)}{c_{n}} \tilde{L}(n-1) \bar{F}^{x_{n-1}}\left(\Delta t_{n}\right) \mathbf{1}_{i} \mathbf{1}_{j}^{\prime} \\
= & \hat{\boldsymbol{m}}_{i j}^{a b}\left(t_{n-1}\right) \frac{f^{x_{n-1} x_{n}}\left(\Delta t_{n}\right)}{c_{n}} \\
& +I_{\left\{x_{n-1}=a, x_{n}=b\right\}} \frac{g_{a b}(i j)}{c_{n}} \tilde{L}(n-1) \bar{F}^{x_{n-1}}\left(\Delta t_{n}\right) \mathbf{1}_{i} \mathbf{1}_{j}^{\prime} .
\end{aligned}
$$

The result (34) then follows by substituting (9) and (10) into (38).

Note that $\tilde{L}(n-1)$ in (34) equals the sum of $\hat{m}_{i j}^{a b}(n-1)$ over all $(a, i) \neq(b, j)$.

\section{Total Sojourn Time Recursion}

The total sojourn time of $Z$ in state $(a, i)$ in the interval $[0, t]$ is given by

$$
D_{i}^{a}(t)=\int_{0}^{t} \chi_{a i}(\tau) d \tau
$$

${ }^{2}$ In the first two lines of [22, Eq. 59], $t_{k}$ should be changed to $t_{k+1}$ in four places. These corrections do not change the end result. and its conditional mean estimate is given by

$$
\hat{D}_{i}^{a}(t)=\int_{0}^{t} P(Z(\tau)=(a, i) \mid X(\xi), 0 \leq \xi \leq t) d \tau .
$$

A causal recursion for $\hat{D}_{i}^{a}(t)$ is obtained from a causal recursion for

$$
\hat{\boldsymbol{D}}_{i}^{a}(t)=E\left\{D_{i}^{a}(t) \boldsymbol{\varphi}(t) \mid X(\tau), 0 \leq \tau \leq t\right\}
$$

using $\hat{D}_{i}^{a}(t)=\hat{\boldsymbol{D}}_{i}^{a}(t) \mathbf{1}$. The $l$ th component of $\hat{\boldsymbol{D}}_{i}^{a}(t)$ is given by

$$
\begin{aligned}
& {\left[\hat{\boldsymbol{D}}_{i}^{a}(t)\right]_{l}} \\
& =\int_{0}^{t} P(Z(\tau)=(a, i), S(t)=l \mid X(\xi), 0 \leq \xi \leq t) d \tau
\end{aligned}
$$

and a causal recursion can be developed by a similar approach to that used in the proof of Proposition 1.

Proposition 3:

$$
\begin{aligned}
\hat{\boldsymbol{D}}_{i}^{a}\left(t_{n}\right)=\frac{1}{c_{n}}\left\{\hat{\boldsymbol{D}}_{i}^{a}\left(t_{n-1}\right) e^{G_{x_{n-1} x_{n-1}} \Delta t_{n}}\right. & \\
& \left.+I_{\left\{x_{n-1}=a\right\}} \mathbf{1}_{i}^{\prime}\left[e^{C_{n-1}^{i} \Delta t_{n}}\right]_{21}\right\} G_{x_{n-1} x_{n}},
\end{aligned}
$$

for $n \geq 1$, and with the initialization $\hat{\boldsymbol{D}}_{i}^{a}\left(t_{0}\right)=\mathbf{0}$.

\section{Remarks}

The non-causal estimators developed in [22] involve a so-called backward variable $\tilde{R}(k)$, which is computed by means of a backward recursion. We remark that the causal and the non-causal estimators for the state, number of jumps, and total sojourn time, respectively, compute precisely the same estimates at time $t_{n}$ when each is based on the same set of observations during $\left[0, t_{n}\right]$. From [22, Eq. (28)], it is easy to see that $\tilde{R}(k)=\tilde{M}(k, n) \mathbf{1}$, assuming the length of the observation interval is $T=t_{n}$. Whereas $\tilde{R}(k)$ is computed using a backward recursion, $\tilde{M}(k, n)$ is computed here, as in [34], using the forward recursion given in (25). A similar approach was used in [36], and references therein, to convert the forward-backward recursions in the Baum-Welch algorithm to forward-only recursions.

The main advantage of the causal recursions is that the observation data does not need to be stored, which yields an online implementation; however, the computational complexity of the causal recursions is higher than that of the non-causal recursions in [22]. The non-causal recursions have a computational complexity of $O\left(r^{3} N\right)$, where $N$ is the number of observations, or equivalently, $O\left(r^{3}\right)$ per jump of the observable process. ${ }^{3}$. By contrast, the causal recursions have a computational complexity of $O\left(r^{4} d^{2}\right)$ per jump. To see this, note that the causal recursions in Propositions 1-3 involve multiplication of a $1 \times r$ row vector by an $r \times r$ matrix, which yields a computational complexity of $O\left(r^{2}\right)$ per jump. The number of vector estimates that need to be computed is dominated by $\hat{\boldsymbol{m}}_{i j}^{a b}\left(t_{n}\right)$ in Proposition 2, i.e., $O\left(r^{2} d^{2}\right)$. This yields a complexity of $O\left(r^{4} d^{2}\right)$. In addition, the number of jumps recursion in Proposition 1 involves

${ }^{3}$ In [22], the complexity of the non-causal recursions was stated to be $O\left(r^{3}+\right.$ $\left.r^{2} d^{2}\right)$, but the term $r^{2} d^{2}$ is for all $N$ jumps. Hence, the actual complexity per jump of the observable process is $O\left(r^{3}\right)$. 
the computation of the matrix exponential $e^{C_{n-1}^{j} \Delta t_{n}}$, which requires $O\left(r^{3}\right)$ arithmetic operations [23], for each $j \in \mathbb{S}_{x_{n-1}}$. Hence, the computational requirement due to the matrix exponentials is $O\left(r^{4}\right)$ per jump, which does not contribute materially to the $O\left(r^{4} d^{2}\right)$ complexity of the number of jumps recursion. We remark that the computation time requirement for the causal recursions can, in principle, be reduced from $O\left(r^{4} d^{2}\right)$ to $O\left(r^{3}\right)$ per step, i.e., the same complexity as the non-causal recursions, by executing the recursions and the computation of the matrix exponentials in parallel.

\section{Causal Recursions for Particular Models}

In this section, we specialize the explicit causal recursions to three particular models of interest. These are the MMMP, the MMPP, and the MAP. In all three cases, the underlying process is an irreducible Markov chain. Hence, $r_{a}=r$ for all $a \in \mathbb{X}$, and the generator of the underlying chain is given by [3]

$$
Q=\left\{q_{i j}\right\}=\sum_{b \in \mathbb{X}} G_{a b}, a \in \mathbb{X} .
$$

\section{A. $M M M P$}

An MMMP is a special bivariate Markov chain $(X, S)$ where $S$ is a Markov chain with generator $Q$, and $X$ given $S$ is a nonhomogeneous Markov chain with generator $G_{i}=\left\{g_{a b}(i), a, b \in \mathbb{X}\right\}$ when $S_{t}=i$. The MMMP may be viewed as a bivariate Markov chain with

$$
\begin{aligned}
G_{a b} & =\operatorname{diag}\left\{g_{a b}(i), i \in \mathbb{S}\right\}, a \neq b ; \\
G_{a a} & =Q-\sum_{b \neq a} G_{a b}, a, b \in \mathbb{X} .
\end{aligned}
$$

The number of components of the generator matrix $G$ that need to be estimated for an MMMP is $r(r-1)+r d(d-1)$, as opposed to $r d(r d-1)$ for the general bivariate Markov chain. For the MMMP process, causal recursions were derived in [11] for the state, number of jumps, and total sojourn time of the underlying Markov chain, as well as for the conditional number of jumps and total sojourn time of the observable process given any state of the underlying Markov chain. We note that in the notation of [11, Eq. (45)], the equivalent of $G_{a a}$ includes an additive diagonal matrix which is eliminated by the scaling procedure. In the remainder of this section we show that these recursions coincide with the more general recursions developed in this paper.

1) State Recursion: The state recursion (13) is equivalent to the scaled version of state recursion [11, Eq. (62)]. In this case, the initial distribution of the sampled underlying Markov chain $\left\{S_{k}\right\}$ is given by $\sum_{a=1}^{d} \nu_{a}$.

2) Number of Jumps Recursion: Let $\hat{m}_{i j}\left(t_{n}\right), i \neq j$, denote the number of jumps of the underlying chain in $\left[0, t_{n}\right]$. Since the underlying and observable chains do not jump simultaneously, $\hat{m}_{i j}\left(t_{n}\right)$ can be expressed in terms of the estimators for the associated bivariate Markov chain as follows:

$$
\hat{m}_{i j}\left(t_{n}\right)=\sum_{a \in \mathbb{X}} \hat{m}_{i j}^{a a}\left(t_{n}\right) .
$$

The corresponding vector estimate is given by

$$
\hat{\boldsymbol{m}}_{i j}\left(t_{n}\right)=\sum_{a \in \mathbb{X}} \hat{\boldsymbol{m}}_{i j}^{a a}\left(t_{n}\right)
$$

Clearly, $\hat{m}_{i j}\left(t_{n}\right)=\hat{\boldsymbol{m}}_{i j}\left(t_{n}\right) \mathbf{1}$. Applying (26) in (47), we have

$$
\begin{aligned}
\hat{\boldsymbol{m}}_{i j}\left(t_{n}\right)= & \frac{1}{c_{n}}\left\{\hat{\boldsymbol{m}}_{i j}\left(t_{n-1}\right) e^{G_{x_{n-1} x_{n-1}} \Delta t_{n}}\right. \\
& \left.+g_{x_{n-1} x_{n-1}}(i j) \mathbf{1}_{i}^{\prime}\left[e^{C_{n-1}^{j} \Delta t_{n}}\right]_{21}\right\} G_{x_{n-1} x_{n}} .
\end{aligned}
$$

Since $g_{a a}(i j)=q_{i j}$ for all $a \in \mathbb{X}$ whenever $i \neq j$,(48) becomes

$$
\begin{array}{r}
\hat{\boldsymbol{m}}_{i j}\left(t_{n}\right)=\frac{1}{c_{n}}\left\{\hat{\boldsymbol{m}}_{i j}\left(t_{n-1}\right) e^{G_{x_{n-1} x_{n-1}} \Delta t_{n}}\right. \\
\left.+q_{i j} \mathbf{1}_{i}^{\prime}\left[e^{C_{n-1}^{j} \Delta t_{n}}\right]_{21}\right\} G_{x_{n-1} x_{n}},
\end{array}
$$

which is equivalent to the scaled version of [11, Eq. (63)].

3) Conditional Number of Jumps Recursion: Let $\hat{m}_{i}^{a b}\left(t_{n}\right)$, $a \neq b$, denote the number of jumps in $\left[0, t_{n}\right]$ of the observable chain from state $a$ to state $b$ while the underlying chain remains in state $i \in \mathbb{S}$. Since the underlying and observable chains of the MMMP do not jump simultaneously, we have $\hat{m}_{i}^{a b}\left(t_{n}\right)=$ $\hat{m}_{i i}^{a b}\left(t_{n}\right)$ for all $i \in \mathbb{S}$ and $\hat{m}_{i j}^{a b}\left(t_{n}\right)=0$ whenever $i \neq j$. The corresponding vector estimate is given by

$$
\hat{\boldsymbol{m}}_{i}^{a b}\left(t_{n}\right)=\hat{\boldsymbol{m}}_{i i}^{a b}\left(t_{n}\right) .
$$

Applying (34), we have

$$
\begin{aligned}
& \hat{\boldsymbol{m}}_{i}^{a b}\left(t_{n}\right)=\frac{1}{c_{n}}\left\{\hat{\boldsymbol{m}}_{i}^{a b}\left(t_{n-1}\right) e^{G_{x_{n-1} x_{n-1}} \Delta t_{n}} G_{x_{n-1} x_{n}}\right. \\
& \left.\quad+I_{\left\{x_{n-1}=a, x_{n}=b\right\}} g_{a b}(i) \tilde{L}(n-1) e^{G_{x_{n-1} x_{n-1}} \Delta t_{n}} \mathbf{1}_{i} \mathbf{1}_{i}^{\prime}\right\} .
\end{aligned}
$$

Noting that

$$
g_{a b}(i) \mathbf{1}_{i} \mathbf{1}_{i}^{\prime}=G_{a b} \mathbf{1}_{i} \mathbf{1}_{i}^{\prime},
$$

we can write (51) as follows:

$$
\begin{aligned}
\hat{\boldsymbol{m}}_{i}^{a b}\left(t_{n}\right)= & \frac{1}{c_{n}} \hat{\boldsymbol{m}}_{i}^{a b}\left(t_{n-1}\right) e^{G_{x_{n-1} x_{n-1}} \Delta t_{n}} G_{x_{n-1} x_{n}} \\
& +I_{\left\{x_{n-1}=a, x_{n}=b\right\}} \frac{\tilde{L}(n-1) e^{G_{x_{n-1} x_{n-1}} \Delta t_{n}} G_{a b}}{c_{n}} \mathbf{1}_{i} \mathbf{1}_{i} \\
= & \frac{1}{c_{n}} \hat{\boldsymbol{m}}_{i}^{a b}\left(t_{n-1}\right) e^{G_{x_{n-1} x_{n-1}} \Delta t_{n}} G_{x_{n-1} x_{n}} \\
& +I_{\left\{x_{n-1}=a, x_{n}=b\right\}} \tilde{L}(n) \mathbf{1}_{i} \mathbf{1}_{i}
\end{aligned}
$$

which is equivalent to the scaled version of [11, Eq. (65) $]^{4}$.

4) Total Sojourn Time Recursion: The total sojourn time recursion (43) is formally equivalent to the scaled version of the recursion for the conditional total sojourn time estimation for the MMMP given in [11, Eq. (66)].

\footnotetext{
${ }^{4}$ In the statement immediately following [11, Eq. (65)] regarding scaling of this recursion, $\pi_{t_{\nu+1}}(\varphi)$ is normalized twice, once when it is replaced by $\tilde{\pi}_{t_{\nu+1}}(\varphi)$ and then when the rhs of (65) is normalized by $c_{\nu+1}$. The correct statement is as follows: "Apply the rhs with $\pi_{t_{\nu}}\left(K_{\bar{l} \bar{n}}^{k} \varphi\right)$ replaced by $\tilde{\pi}_{t_{\nu}}\left(K_{\bar{l} \bar{n}}^{k} \varphi\right) / c_{\nu+1}$ and $\pi_{t_{\nu+1}}(\varphi)$ replaced by $\tilde{\pi}_{t_{\nu+1}}(\varphi)$ to obtain $\tilde{\pi}_{t_{\nu+1}}\left(K_{\bar{l} \bar{n}}^{k} \varphi\right)$." Numerical implementation of [11, Eq. (65)] was done correctly, as indicated here.
} 
5) Conditional Total Sojourn Time Recursion: The conditional mean estimate of the total sojourn time of the bivariate Markov chain in an underlying state $i \in \mathbb{S}$ is given by

$$
\hat{D}_{i}\left(t_{n}\right)=\sum_{a \in \mathbb{X}} \hat{D}_{i}^{a}\left(t_{n}\right)
$$

The corresponding vector estimate is given by

$$
\hat{\boldsymbol{D}}_{i}\left(t_{n}\right)=\sum_{a \in \mathbb{X}} \hat{\boldsymbol{D}}_{i}^{a}\left(t_{n}\right)
$$

Clearly, $\hat{D}_{i}\left(t_{n}\right)=\hat{\boldsymbol{D}}_{i}\left(t_{n}\right) \mathbf{1}$. Applying (43) in (55), we have

$$
\begin{aligned}
\hat{\boldsymbol{D}}_{i}\left(t_{n}\right)=\frac{1}{c_{n}}\left\{\hat{\boldsymbol{D}}_{i}\left(t_{n-1}\right) e^{G_{x_{n-1} x_{n-1}} \Delta t_{n}}\right. & \\
& \left.+\mathbf{1}_{i}\left[e^{C_{n-1}^{i} \Delta t_{n}}\right]_{21}\right\} G_{x_{n-1} x_{n}},
\end{aligned}
$$

which is equivalent to the recursion [11, Eq. (64)] after scaling is applied.

\section{B. $M M P P$}

The MMPP may be viewed as a special bivariate Markov chain with $d=2, G_{12}=G_{21}$, and $G_{11}=G_{22}$. When the conditional Poisson rates of the MMPP are given by the diagonal terms of $\Lambda=\operatorname{diag}\left(\lambda_{1}, \ldots, \lambda_{r}\right)$, then $G_{12}=\Lambda$ and $G_{11}=Q-\Lambda$. Note that the number of components that need to be estimated in $G$ for the MMPP is $r^{2}$. The causal recursions for the MMMP apply straightforwardly to this case. Clearly, $I\left(x_{n-1}=a, x_{n}=b\right)=1$ for the conditional number of jumps recursion (53).

\section{MAP}

As discussed in [22, Section 2.4], the MAP may be represented using a finite-state bivariate Markov chain $Z=(X, S)$, where $X$ represents the modulo-2 counts of the observable process. Similarly to the MMPP, $d=2, G_{12}=G_{21}$, and $G_{11}=G_{22}$. Here, however, $G_{12}$ may be any rate matrix, and $X$ and $S$ may jump simultaneously. A generalization of the MAP is given by the BMAP for which the observable process may have multiple arrivals at each arrival epoch. The BMAP has a similar representation as a finite-state bivariate Markov chain [22, Eq. (40)]. In the remainder of this section, we shall focus on causal recursions for the MAP, but recursions could also be developed straightforwardly for the BMAP using the same approach. The parameter of the MAP is given by $\left(H_{0}, H_{1}\right)$, where $H_{0}=G_{11}$ and $H_{1}=G_{12}$; $H_{a}=\left\{h_{a}(i j), i, j=1, \ldots, r\right\}$ for $a=0,1$.

1) State Recursion: The state recursion follows straightforwardly from (13) with the initial distribution of the underlying Markov chain given by $\sum_{a=1}^{r} \nu_{a}$.

2) Number of Jumps Recursion: Let $\hat{m}_{i j}^{(0)}\left(t_{n}\right), i \neq j$, denote the number of jumps in $\left[0, t_{n}\right]$ of the underlying chain that do not coincide with a jump of the observable process. In terms of the estimators for the associated bivariate Markov chain, we have

$$
\hat{m}_{i j}^{(0)}\left(t_{n}\right)=\hat{m}_{i j}^{00}\left(t_{n}\right)+\hat{m}_{i j}^{11}\left(t_{n}\right) .
$$

Next define the corresponding vector estimate

$$
\hat{\boldsymbol{m}}_{i j}^{(0)}\left(t_{n}\right)=\hat{\boldsymbol{m}}_{i j}^{00}\left(t_{n}\right)+\hat{\boldsymbol{m}}_{i j}^{11}\left(t_{n}\right) .
$$

Clearly, $\hat{m}_{i j}^{(0)}\left(t_{n}\right)=\hat{\boldsymbol{m}}_{i j}^{(0)}\left(t_{n}\right) \mathbf{1}$. Applying (26) in (58), we obtain the causal recursion

$$
\begin{aligned}
\hat{\boldsymbol{m}}_{i j}^{(0)}\left(t_{n}\right)=\frac{1}{c_{n}}\{ & \hat{\boldsymbol{m}}_{i j}^{(0)}\left(t_{n-1}\right) e^{H_{0} \Delta t_{n}} \\
& \left.+h_{0}(i j) \mathbf{1}_{i}^{\prime}\left[e^{C_{n-1}^{j} \Delta t_{n}}\right]_{21}\right\} H_{1}, i \neq j .
\end{aligned}
$$

For the MAP, the matrix $C_{k}^{j}$ has the form

$$
C_{k}^{j}=\left[\begin{array}{cc}
H_{0} & \mathbf{0} \\
\tilde{L}(k)^{\prime} \mathbf{1}_{j}^{\prime} & H_{0}^{\prime}
\end{array}\right], j=1, \ldots, r .
$$

Let $\hat{m}_{i j}^{(1)}\left(t_{n}\right)$, denote the number of jumps in $\left[0, t_{n}\right]$ of the observable process which may possibly coincide with a jump of the observable process. In this case,

$$
\hat{m}_{i j}^{(1)}\left(t_{n}\right)=\hat{m}_{i j}^{01}\left(t_{n}\right)+\hat{m}_{i j}^{10}\left(t_{n}\right) .
$$

Define

$$
\hat{\boldsymbol{m}}_{i j}^{(1)}\left(t_{n}\right)=\hat{\boldsymbol{m}}_{i j}^{01}\left(t_{n}\right)+\hat{\boldsymbol{m}}_{i j}^{10}\left(t_{n}\right) .
$$

Clearly, $\hat{m}_{i j}^{(1)}\left(t_{n}\right)=\hat{\boldsymbol{m}}_{i j}^{(1)}\left(t_{n}\right) \mathbf{1}$. Applying (34) in (62), we obtain the causal recursion

$$
\begin{aligned}
\hat{\boldsymbol{m}}_{i j}^{(1)}\left(t_{n}\right)=\frac{1}{c_{n}}\left\{\hat{\boldsymbol{m}}_{i j}^{(1)}\left(t_{n-1}\right) e^{H_{0} \Delta t_{n}} H_{1}\right. & \\
& \left.+h_{1}(i j) \tilde{L}(n-1) e^{H_{0} \Delta t_{n}} \mathbf{1}_{i} \mathbf{1}_{j}^{\prime}\right\},
\end{aligned}
$$

for $i, j=1, \ldots, r$.

3) Total Sojourn Time Recursion: Let $\hat{D}_{i}\left(t_{n}\right)$ denote the total sojourn time in $\left[0, t_{n}\right]$ of the underlying chain in state $i$. Then we have

$$
\hat{D}_{i}\left(t_{n}\right)=\hat{D}_{i}^{0}\left(t_{n}\right)+\hat{D}_{i}^{1}\left(t_{n}\right) .
$$

The corresponding vector estimate is given by

$$
\hat{\boldsymbol{D}}_{i}\left(t_{n}\right)=\hat{\boldsymbol{D}}_{i}^{0}\left(t_{n}\right)+\hat{\boldsymbol{D}}_{i}^{1}\left(t_{n}\right) .
$$

Clearly, $\hat{D}_{i}\left(t_{n}\right)=\hat{\boldsymbol{D}}_{i}\left(t_{n}\right) \mathbf{1}$. Applying the causal recursion (43) to the right-hand side of (65), we obtain

$$
\hat{\boldsymbol{D}}_{i}\left(t_{n}\right)=\frac{1}{c_{n}}\left\{\hat{\boldsymbol{D}}_{i}\left(t_{n-1}\right) e^{H_{0} \Delta t_{n}}+\mathbf{1}_{i}^{\prime}\left[e^{C_{n-1}^{i} \Delta t_{n}}\right]_{21}\right\} H_{1},
$$

$i=1, \ldots, r$, where $C_{n}^{i}$ has the form given in (60).

\section{NUMERICAL EXAMPLE}

In this section, we demonstrate the performance of the causal recursions (27), (34), and (43) for estimating the number of jumps and total sojourn time statistics of a bivariate Markov 


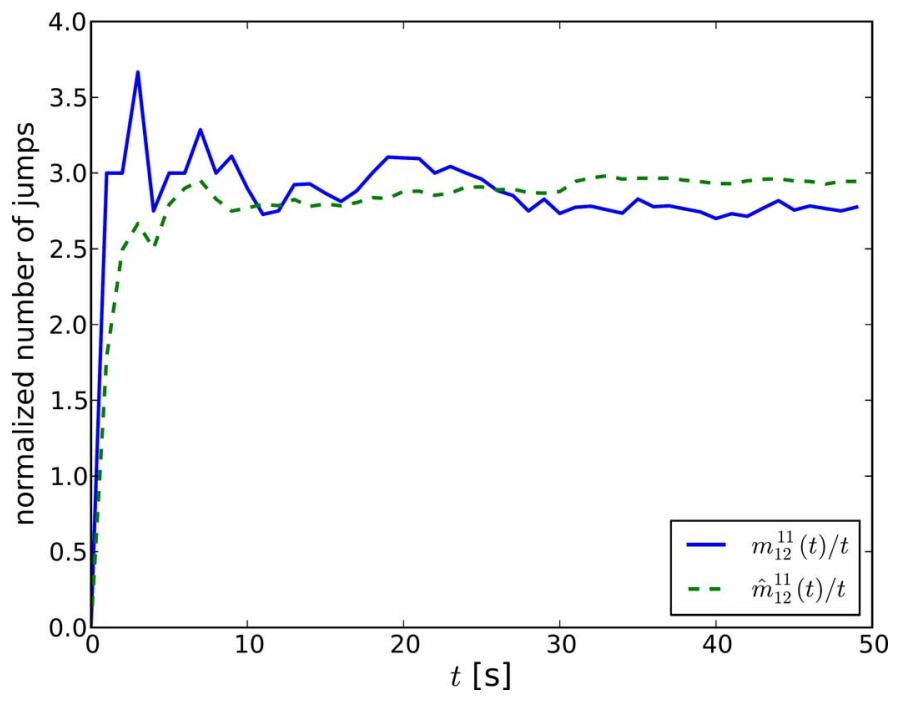

Fig. 1. Normalized number of jumps, $m_{12}^{11}(t) / t$, and the conditional mean estimate $\hat{m}_{12}^{11}(t) / t$.

chain. The recursions were implemented in Python using the SciPy and NumPy libraries.

We consider the example of a bivariate Markov chain from [22] with generator given by

$$
G=\left[\begin{array}{rr|rr}
-70 & 10 & 50 & 10 \\
20 & -55 & 25 & 10 \\
\hline 50 & 0 & -60 & 10 \\
0 & 10 & 20 & -30
\end{array}\right]=\left[\begin{array}{ll}
G_{11} & G_{12} \\
G_{21} & G_{22}
\end{array}\right]
$$

Note that the bivariate Markov chain represented by this generator is not an MMMP, since $G_{12}$ is not a diagonal matrix, nor is it a MAP, since $G_{12} \neq G_{21}$. To demonstrate the performance of the causal recursions, a realization of the bivariate Markov chain $Z=(X, S)$ consisting of $M+1$ jumps of the observable process $X$, including the initial jump at time $t=0$, was generated. The corresponding observable sequence can be characterized by $\left\{\left(x_{k}, t_{k}\right)\right\}_{k=0}^{M}$, where $t_{k}$ denotes the time of the $k$ th jump and $x_{k}$ is the state of $X$ just after time $t_{k}$. For this example, we have set $M=2000$.

Fig. 1 shows, for a single sample path of the bivariate Markov chain, a plot of the normalized number of jumps from state $(1,1)$ to state $(1,2)$, i.e., $m_{12}^{11}(t) / t$, obtained from the generated data, and its estimate, $\hat{m}_{12}^{11}(t) / t$, obtained from Proposition 1, at the observable jump times $t_{n}, n=1,2, \ldots$. From the figure, we see that the conditional mean estimate of the normalized number of jumps follow closely the actual normalized number of jumps. Similar behavior can be observed for normalized versions of the other estimates $\hat{m}_{i j}^{a b}(t)$ and $\hat{D}_{i}^{a}(t)$ for different values of $(a, i)$, $(b, j) \in \mathbb{Z}$.

To provide a more quantitative sense of the performance of the estimators, we computed the empirical mean squared error (MSE) for the normalized statistics. Figs. 2-4 show the empirical MSE for the normalized estimates $\hat{m}_{12}^{11}(t) / t, \hat{m}_{11}^{12}(t) / t$, and $\hat{D}_{1}^{1}(t) / t$, respectively. In each case, 50 sample paths of the bivariate Markov chain were generated and $95 \%$ confidence intervals were computed, as shown in the figures. For each of the

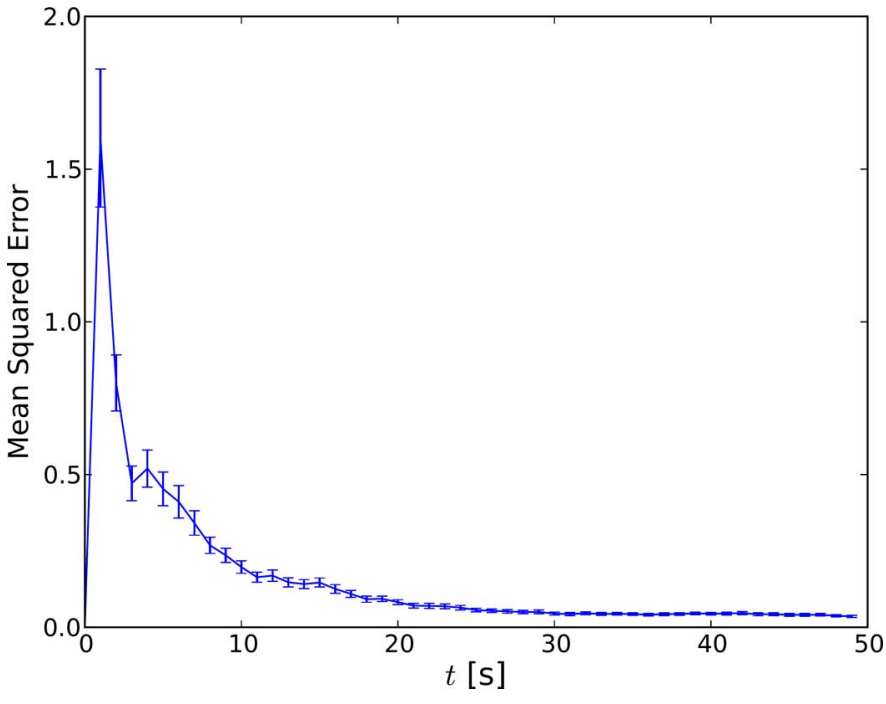

Fig. 2. MSE for $\hat{m}_{12}^{11}(t) / t$.

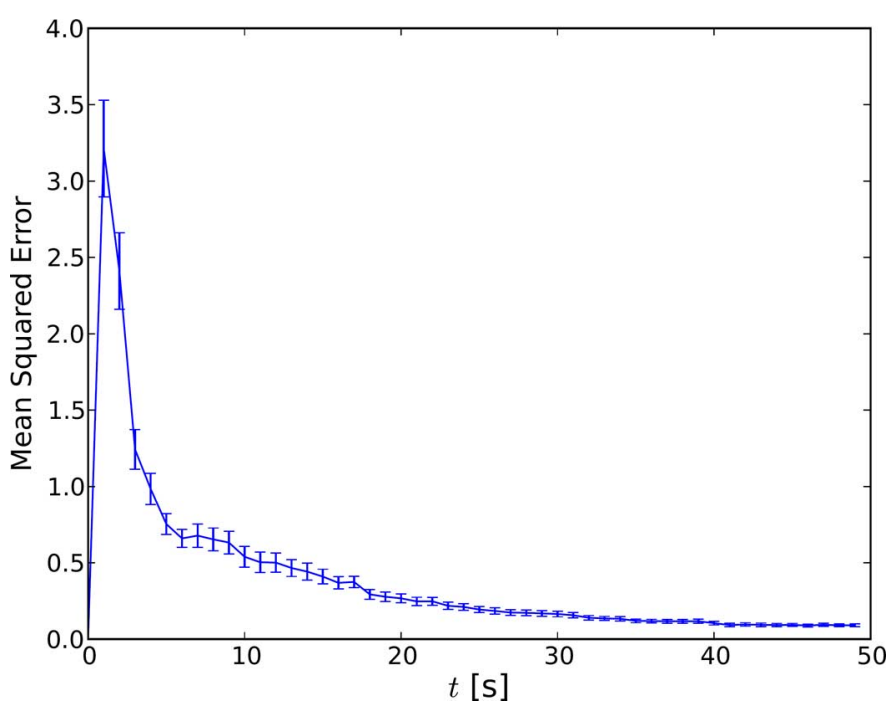

Fig. 3. MSE for $\hat{m}_{11}^{12}(t) / t$.

normalized estimates, the MSE converges rather quickly to a small positive value. Similar performance was observed with other examples of bivariate Markov chains. It should be emphasized that the mean squared errors in estimating the number of jumps and the total sojourn time by the causal estimators of this paper eventually achieve the performance of the fixed-interval smoothed estimates of [22] when the observations have been exhausted.

We remark that for a fixed time $t$, the sum of the total sojourn times $D_{i}(t)$ over $i \in \mathbb{S}$ equals $t$. Similarly, $\sum_{i \in \mathbb{S}} \hat{D}_{i}(t)=t$. Therefore, the error in a particular total sojourn time estimate $\hat{D}_{i}(t)$ is upper-bounded by $t$. On the other hand, the number of jumps, $m_{i j}(t)$, is unbounded for fixed $t$; hence, the error in the number of jumps estimate, $\hat{m}_{i j}(t)$, is unbounded. This accounts for the significantly larger MSE results observed for the estimated number of jumps in Figs. 2 and 3 compared to the results for the total sojourn time in Fig. 4. 


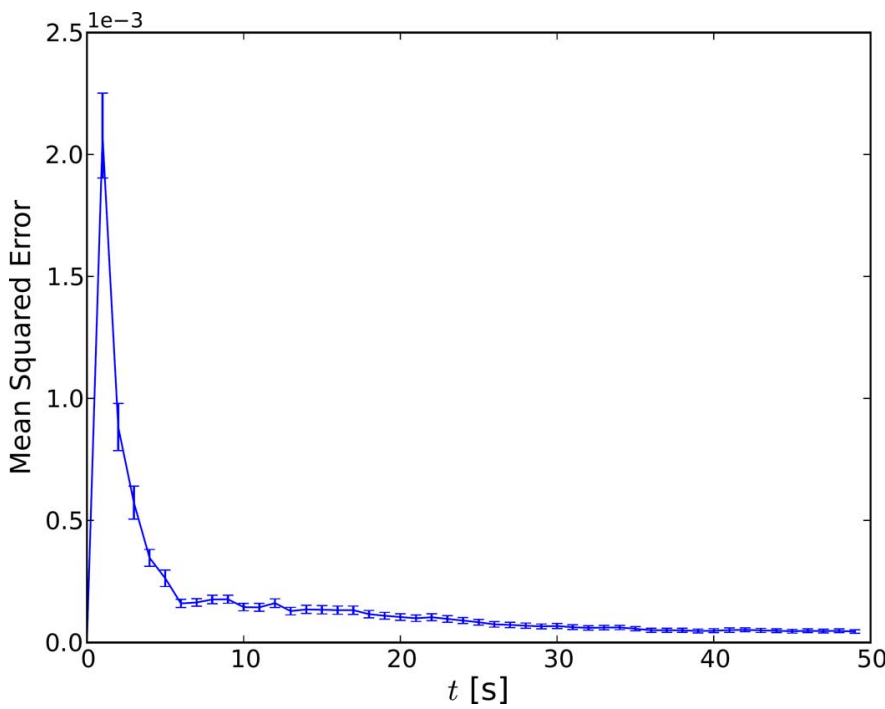

Fig. 4. MSE for $\hat{D}_{1}^{1}(t) / t$.

\section{CONCLUSION}

We have derived explicit causal recursions for estimating the state, the number of jumps, and the total sojourn time for a bivariate Markov chain. Previously, non-causal recursions were derived for these statistics in [22]. The explicit state recursion was originally developed in [30], [39]. In addition, explicit causal recursions for the MMPP and the MMMP were developed in [11] using a different approach which relies on transformation of measure. We specialized our recursions for the MMMP, its the special case the MMPP, and the MAP, and showed that the recursions for the MMMP coincide with the recursions in [11]. Using the same approach, similar recursions can be developed readily for the batch Markovian arrival process (BMAP). The approach is also applicable to multivariate Markov chains, which are commonly used in ion-channel current modeling [3]. We have also demonstrated the performance of the causal estimators with a numerical example.

\section{ACKNOWLEDGMENT}

The authors would like to thank the anonymous reviewers for their comments, which helped to improve the presentation of this paper.

\section{REFERENCES}

[1] A. Albert, "Estimating the infinitesimal generator of a continuous time, finite state Markov process," Ann. Math. Stat., vol. 33, pp. 727-753, 1962.

[2] F. Ball, R. K. Milne, and G. F. Yeo, "Continuous-time Markov chains in a random environment, with applications to ion channel modelling," Adv. Appl. Probab., vol. 26, pp. 919-946, 1994.

[3] F. Ball and G. F. Yeo, "Lumpability and marginalisability for continuous time Markov chains," J. Appl. Prob., vol. 30, no. 3, pp. 518-528, 1993.

[4] F. G. Ball and J. A. Rice, "Stochastic models for ion channels: Introduction and bibliography," Math. Biosci., vol. 112, pp. 189-206, 1992.

[5] L. Breuer, "An EM algorithm for batch Markovian arrival processes and its comparison to a simpler estimation procedure," Ann. Oper. Res., vol. 112, no. 1-4, pp. 123-138, 2002.

[6] D. Bryant, N. Galtier, and M. A. Poursat, "Likelihood calculation in molecular phylogenetics," in Mathematics of Evolution and Phylogeny, O. Gascuel Ed ed. London, U.K.: Oxford Univ. Press, 2007.

[7] C. J. Burke and M. Rosenblatt, "A Markovian function of a Markov chain," Ann. Math. Statist., vol. 29, pp. 1112-1122, 1958.
[8] D. Colquhoun and A. G. Hawkes, "On the stochastic properties of bursts of single ion channel openings and of clusters of bursts," Phil. Trans. R. Soc. London B, vol. 300, no. 1098, pp. 1-59, 1982.

[9] R. J. Elliott, L. Aggoun, and J. B. Moore, Hidden Markov models: Estimation and control. Berlin, Germany: Springer-Verlag, 1997.

[10] R. J. Elliott and W. P. Malcolm, "Discrete-time expectation maximization algorithms for Markov-modulated Poisson processes," IEEE Trans. Autom. Control, vol. 53, no. 1, pp. 247-256, Feb. 2008.

[11] Y. Ephraim and B. L. Mark, "Explicit forward recursive estimators for Markov modulated Markov processes," Stochastic Models, vol. 28, no. 3, pp. 359-387, 2012.

[12] Y. Ephraim and B. L. Mark, "On forward recursive estimation for bivariate Markov chains," in Proc. Conf. Inf. Sci. Syst. (CISS), Princeton, NJ, USA, Mar. 2012.

[13] Y. Ephraim and B. L. Mark, "Bivariate Markov processes and their estimation," Foundations Trends Signal Process., vol. 6, no. 1, pp. 1-95, 2013.

[14] Y. Ephraim and B. L. Mark, "Consistency of maximum likelihood parameter estimation for bivariate Markov chains," Stochastic Models, vol. 29, no. 1, pp. 89-111, 2013.

[15] Y. Ephraim and N. Merhav, "Hidden Markov processes," IEEE Trans. Inf. Theory, vol. 48, no. 6, pp. 1518-1569, Jun. 2002.

[16] Y. Ephraim and W. J. J. Roberts, "An EM algorithm for Markov modulated Markov processes," IEEE Trans. Signal Process., vol. 57, no. 2, pp. 463-470, Feb. 2009.

[17] W. Fischer and K. Meier-Hellstern, "The Markov-modulated Poisson process (MMPP) cookbook," Perform. Eval., vol. 18, pp. 149-171, 1992.

[18] N. Galtier and A. Jean-Marie, "Markov-modulated Markov processes and the covarion process of molecular evolution," J. Comput. Biol., vol. 11, no. 4, pp. 727-733, 2004.

[19] S. Geirhofer, L. Tong, and B. M. Sadler, "Dynamic spectrum access in the time domain: Modeling and exploiting white space," IEEE Commun. Mag., vol. 45, no. 5, pp. 66-72, May 2007.

[20] A. Klemm, C. Lindemann, and M. Lohmann, "Modeling IP traffic using the batch Markovian arrival process," Perform. Eval., vol. 54, pp. 149-173, 2003.

[21] D. M. Lucantoni, "New results on the single server queue with a batch Markovian arrival process," Stochastic Models, vol. 7, no. 1, pp. 1-46, 1991.

[22] B. L. Mark and Y. Ephraim, "An EM algorithm for continuous-time bivariate Markov chains," Comput. Statist. Data Anal., vol. 57, pp. 504-517, 2013.

[23] C. Moler and C. Van Loan, "Nineteen dubious ways to compute the exponential of a matrix, twenty five years later," SIAM Rev., vol. 45, pp. 3-49, 2003.

[24] T. Nguyen, B. L. Mark, and Y. Ephraim, "Hidden Markov process based dynamic spectrum access for cognitive radio," in Proc. Conf. Inf. Sci. Syst. (CISS), Baltimore, MD, USA, Mar. 2011.

[25] T. Nguyen, B. L. Mark, and Y. Ephraim, "Spectrum sensing using a hidden bivariate Markov model," IEEE Trans. Wireless Commun., vol. 12, no. 9, pp. 4582-4591, Sep. 2013.

[26] L. Pachter and B. Sturmfels, "The mathematics of phylogenomics," SIAM Rev., vol. 49, no. 1, pp. 3-31, Mar. 2007.

[27] F. Qin, A. Auerbach, and F. Sachs, "Maximum likelihood estimation of aggregated Markov processes," Proc. R. Soc. London B, vol. 264, pp. 375-383, 1997.

[28] W. J. J. Roberts and Y. Ephraim, "An EM algorithm for ion-channel current estimation," IEEE Trans. Signal Process., vol. 56, no. 1, pp. 26-33, Jan. 2008.

[29] W. J. J. Roberts, Y. Ephraim, and E. Dieguez, “On Rydén's EM algorithm for estimating MMPPs," IEEE Signal Process. Lett., vol. 13, no. 6, pp. 373-377, Jun. 2006.

[30] M. Rudemo, "State estimation for partially observed Markov chains," J. Math. Anal. Appl., vol. 44, pp. 581-611, 1973.

[31] T. Rydén, "Parameter estimation for Markov modulated Poisson processes," Commun. Statist. Stoch. Models, vol. 10, no. 4, pp. 795-829, 1994.

[32] T. Rydén, "An EM algorithm for estimation in Markov-modulated Poisson processes," Comput. Statist. Data Anal., vol. 21, pp. 431-447, 1996.

[33] K. Salamatian and S. Vaton, "Hidden Markov modeling for network communication channels," Proc. ACM Sigmetrics'01, pp. 92-101, Jun. 2001.

[34] C. Stiller and G. Radons, "Online estimation of hidden Markov models," IEEE Signal Process. Lett., vol. 6, no. 8, pp. 213-215, Aug. 1999.

[35] Z. Sun, G. J. Bradford, and J. N. Laneman, "Sequence detection algorithms for PHY-layer sensing in dynamic spectrum access networks," IEEE J. Sel. Topics Signal Process., vol. 5, no. 1, pp. 97-109, Feb. 2011.

[36] W. Turin, "Unidirectional and parallel Baum-Welch algorithms," IEEE Trans. Speech Audio Process., vol. 6, no. 6, pp. 516-523, Nov. 1998. 
[37] C. F. Van Loan, "Computing integrals involving the matrix exponential," IEEE Trans. Autom. Control, vol. 23, no. 3, pp. 395-404, Jun 1978.

[38] W. Wei, B. Wang, and D. Towsley, "Continuous-time hidden Markoy models for network performance evaluation," Perform. Eval., vol. 49, pp. 129-146, Sep. 2002.

[39] A. I. Yashin, "Filtering of jump processes," Automat. Remote Contr. vol. 31 , pp. 725-730, 1970 .

[40] O. Zeitouni and A. Dembo, "Exact filters for the estimation of the number of transitions of finite-state continuous-time Markov processes," IEEE Trans. Inf. Theory, vol. 34, no. 4, pp. 890-893, Apr. 1988.

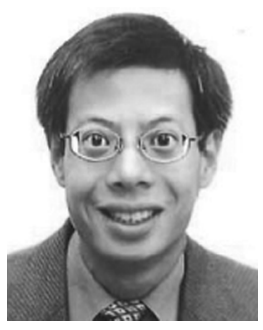

Brian L. Mark (S'91-M'95-SM'08) received the Ph.D. in Electrical Engineering from Princeton University, in 1995 and the B.A.Sc. degree in Computer Engineering with an option in Mathematics from the University of Waterloo in 1991 . He was a Research Staff Member at the C\&C Research Laboratories, NEC USA, from 1995 to 1999. In 1999, he was on part-time leave from NEC as a visiting researcher at Ecole Nationale Supérieure des Télécommunications in Paris, France. In 2000, he joined George Mason University, where he is currently Professor of Electrical and Computer Engineering. His main research interests lie in the design and performance analysis of communication networks. He served as an Associate Editor for IEEE Transactions on Vehicular Technology from 2006-2009.

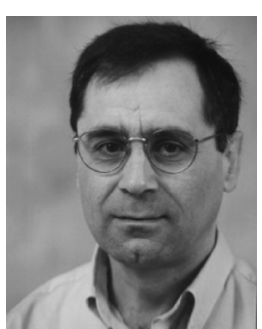

Yariv Ephraim (S'82-M'84-SM'90-F'94) received the B.Sc., M.Sc. and D.Sc. degrees in electrical engineering from the Technion-Israel Institute of Technology, Haifa, Israel, in 1977, 1979, and 1984, respectively. During 1984-1985, he was a Rothschild Postdoctoral Fellow at the Information Systems Laboratory of Stanford University, Stanford, CA. From 1985 to 1993, he was a Member of Technical Staff at the Information Principles Research Laboratory of AT\&T Bell Laboratories, Murray Hill, NJ. In 1991, he joined George Mason University, Fairfax, VA, where he currently is Professor of Electrical and Computer Engineering. Dr. Ephraim served as an Associate Editor of the IEEE Transactions on Audio, Speech, and Language Processing. He is on the Editorial Board of Foundations and Trends in Signal Processing. His research interests are in statistical signal processing. 